\title{
Integrative analysis of the contribution of mRNAs and long non-coding RNAs to the pathogenesis of asthma
}

\author{
XIAOCHUANG LIU $^{1 *}$, YANYAN ZHANG ${ }^{2 *}$, HUI JIANG ${ }^{1}$, NANNAN JIANG $^{3}$ and JIARONG GAO ${ }^{1}$ \\ ${ }^{1}$ Department of Pharmacy, The First Affiliated Hospital of Anhui University of Chinese Medicine, Hefei, Anhui 230031; \\ ${ }^{2}$ Department of Pharmacy, Anhui Medical College, Hefei, Anhui 230601; ${ }^{3}$ Department of Pharmacy, \\ Anhui University of Chinese Medicine, Hefei, Anhui 230012, P.R. China
}

Received November 13, 2018; Accepted May 23, 2019

DOI: $10.3892 / \mathrm{mmr} .2019 .10511$

\begin{abstract}
Asthma, a common but poorly controlled disease, is one of the most serious health problems worldwide; however, the mechanisms underlying the development of asthma remain unknown. Long non-coding RNAs (lncRNAs) and mRNAs serve important roles in the initiation and progression of various diseases. The present study aimed to investigate the role of differentially expressed lncRNAs and mRNAs associated with asthma. Differentially expressed lncRNAs and mRNAs were screened between the expression data of 62 patients with asthma and 43 healthy controls. Gene ontology (GO) and Kyoto Encyclopedia of Genes and Genomes (KEGG) analyses were performed to investigate the biological functions and pathways associated with the lncRNAs and mRNAs identified. Protein-protein interaction (PPI) networks were subsequently generated. In addition, IncRNA-mRNA weighted co-expression networks were obtained. In total, 159 differentially expressed lncRNAs and 1,261 mRNAs were identified. GO and KEGG analyses revealed that differentially expressed mRNAs regulated asthma by participating in the 'vascular endothelial (VEGF) signaling pathway', 'oxidative phosphorylation', 'Fc $\varepsilon$ RI signaling pathway', 'amino sugar and nucleotide sugar metabolism', 'histidine metabolism', ' $\beta$-alanine metabolism' and 'extracellular matrix-receptor interaction' $(\mathrm{P}<0.05)$. Furthermore, protein kinase B 1 had the highest connectivity degree in the PPI network, and was significantly enriched in the 'VEGF signaling pathway' and 'Fc $\varepsilon$ RI signaling pathway'. A total of 8 lncRNAs in the lncRNA-mRNA co-expression network were reported to
\end{abstract}

Correspondence to: Professor Jiarong Gao, Department of Pharmacy, The First Affiliated Hospital of Anhui University of Chinese Medicine, 117 Meishan Road, Hefei, Anhui 230031, P.R. China

E-mail: zyfygjr2006@163.com

*Contributed equally

Key words: asthma, long non-coding RNAs, gene ontology, mechanisms, pathways interact with 52 differentially expressed genes, which were enriched in asthma-associated GO and KEGG pathways. The results obtained in the present study may provide insight into the profile of differentially expressed lncRNAs associated with asthma. The identification of a cluster of dysregulated lncRNAs and mRNAs may serve as a potential therapeutic strategy to reverse the progression of asthma.

\section{Introduction}

Asthma is a chronic inflammatory disease of the airways, characterized by airway hyperresponsiveness, chronic lung inflammation and airway remodeling $(1,2)$. Asthma affects an estimated 358 million people worldwide, leading to a mortality rate of 0.4 million in 2015. Inhaled corticosteroids are the main method of pharmaceutical treatment; however, their effects are not satisfactory. Smoking and occupational asthmagens are the primary risk factors for asthma (3). Asthma affects people of all ages and there are no effective clinical treatments due to the complex pathophysiology of the condition (4). Therefore, understanding the molecular basis underlying the pathophysiology of asthma, and the screening of molecular markers for the development of therapeutic targets remains critical.

Long non-coding RNAs (lncRNAs) are RNA transcripts $>200$ nucleotides in length (5). The various functions of lncRNAs have been reported with advances in sequencing and bioinformatics analysis (6). Previous studies have suggested that IncRNAs regulate gene expression by interacting with DNA, RNA or proteins $(5,6)$. For example, the lncRNA MAR1 acts as a sponge for miR-487b, promoting skeletal muscle differentiation and regeneration (7). Thus, these transcripts may be considered as potential biomarkers and therapeutic targets (8-12); however, the expression profiles of IncRNAs and mRNAs in asthma remain unclear.

In the present study, microarray data was downloaded from the National Center of Biotechnology Information (NCBI) Gene Expression Omnibus (GEO) database for the analysis of differentially expressed mRNA and lncRNA profiles in asthma. In addition, the database was used to investigate asthma-associated mRNAs and lncRNAs in airway epithelial brushings. Gene Ontology (GO), Kyoto Encyclopedia of Genes and Genomes (KEGG), protein-protein interaction (PPI) network and weighted correlation network 
analyses (WGCNAs) were applied to investigate the role of the differentially expressed lncRNAs and mRNAs in asthma. To the best of our knowledge, the present study is the first to comprehensively analyze mRNAs and lncRNAs in asthma. The findings may provide in-depth molecular insight into the pathophysiology of this condition.

\section{Materials and methods}

Tissue samples and data acquisition. The gene expression data of the GSE67472 dataset was downloaded from the NCBI GEO (www.ncbi.nlm.nih.gov/geo). The GEO is the largest database for high-throughput molecular data, primarily gene expression data (13), and contains links to $\sim 20,000$ published studies comprising 800,000 samples derived from $>1,600$ organisms (14). Following screening, the GSE67472 dataset with a large sample size was selected for analysis to ensure the stability and reliability of the data (15). Analysis was conducted via the GPL1355 platform of Affymetrix Human Genome U133 Plus 2.0 (Thermo Fisher Scientific, Inc.). The GSE67472 dataset containing the data of airway epithelial gene expression in 62 patients with asthma and 43 healthy control samples from airway epithelial brushings was included in the microarray analysis (16).

GeneChip probe re-annotation. Numerous lncRNAs were identified via the Affymetrix microarray according to the lncRNA classification pipeline developed in a previous study (17). The latest version of NetAffx Annotation File (release 36; HG-U133_Plus_2 Annotations; CSV format; $36 \mathrm{MB}$; accessed on 7th December 2016) was obtained from Affymetrix (Thermo Fisher Scientific, Inc.). This annotation file was mapped to the identifications (IDs) of the HG-U133_Plus_2 probe sets. For the probe sets from the RefSeq database (RefSeq Release 89; www.ncbi. nlm.nih.gov/refseq/), probes labeled with protein mixed (NP) were removed, but those with an ID beginning with non-coding RNAs (NR) were included. For the probe sets from the Ensembl database (ensemble 96; www.ensembl. org/index.html), the online software BioMart (GRCh38. p11; http://asia.ensembl.org/biomart/martview/f0b2ccb5ee23510bf3f1e71d87ba7122) was applied to convert Affymetrix microarray IDs to Ensembl IDs with the corresponding gene type. Probe sets from NONCODE were retained. Furthermore, genes annotated as 'lincRNA', 'sense_intronic', 'processed_transcript', 'antisense', 'sense_overlapping', ' 3 prime_overlapping_ncRNA' or ' $m i s c \_R N A$ ' were retained. Finally, probe set IDs annotated as 'microRNA' or 'snoRNAs', and other small RNAs were removed.

Data preprocessing. Affymetrix Expression Console (v1.4; http://www.affymetrix.com/support/technical/byproduct.affx? product=expressionconsole) with Ro-bust microarray was applied to normalize the raw files.Limma package (http://bioinf. wehi.edu.au/limma) in R (version 3.5.1; https://cran.r-project. org/) was used to identify differentially expressed lncRNAs and mRNAs among the asthma and control groups via a t-test (18). Fold change (FC) $>1.2$ and $\mathrm{P}<0.05$ were set as the criteria for differential expression (19). Hierarchical clustering was conducted in $\mathrm{R}$ scripts.
GO and pathway enrichment analysis. GO (geneontology. org) analysis, frequently used in functional enrichment studies of large-scale genes (20), and KEGG (www.genome. $\mathrm{jp} / \mathrm{kegg}$ ) enrichment analysis were performed to investigate the biological pathways that involve differentially expressed mRNAs. In the present study, clusterProfiler (v3.12.0; guangchuangyu.github.io/software/clusterProfiler) and Database for Annotation, Visualization and Integrated Discovery tools (v6.8; https://david.ncifcrf.gov/) were used to analyze the functional enrichment conditions for dysregulated mRNAs (21-23). The false discovery rate (FDR) was calculated to correct the P-value and FDR $<0.05$ was selected as the threshold for a statistically significant difference.

PPI network construction. Protein interactions were analyzed using the online Search Tool for the Retrieval of Interacting Genes (v 10.5; string-db.org) tool and a combined score of $>0.7$ was used as the cut-off criterion (24-26). PPI networks were subsequently generated using Cytoscape software (v3.2.8; cytoscapeweb.cytoscape.org) $(27,28)$.

Construction of the IncRNA-mRNA WGCNA. The WGCNA package (29) in $\mathrm{R}$ was used to construct the co-expression network of lncRNAs and mRNAs as follows: i) Network construction, the weighted co-expression network of lncRNAs and mRNAs was specified in its adjacency matrix $\left(\mathrm{a}_{\mathrm{mn}}\right)$, which encodes the network connection strength between nodes $\mathrm{m}$ and $\mathrm{n}$. In order to calculate the $\mathrm{a}_{\mathrm{mn}}$, the default approach was employed, which defines the co-expression similarity $\left(\mathrm{S}_{\mathrm{mn}}\right)$ as the absolute value of the correlation coefficient between the nodes of $\mathrm{m}$ and $\mathrm{n}$, and $\mathrm{S}_{\mathrm{mn}}=\left|\operatorname{cor}\left(\mathrm{a}_{\mathrm{m}}, \mathrm{a}_{\mathrm{n}}\right)\right|$. The weighted adjacency $\mathrm{a}_{\mathrm{mn}}$ between two genes is proportional to their similarity on a logarithmic scale with absolute value of the correlation to a power $\beta \geq 0.8, \log \left(\mathrm{a}_{\mathrm{mn}}\right)=\beta \times \log \left(\mathrm{S}_{\mathrm{mn}}\right)$. Adjacency functions were obtained by using the approximate scale-free topology criterion (30). The $\mathrm{a}_{\mathrm{mn}}$ was converted into a topology matrix; and ii) module detection: The Dynamic Tree Cut and Static Tree Cut methods were applied to detect modules with $\geq 30$ lncRNAs/genes (31). The cluster dendrogram was visualization using the WGCNA package.

\section{Results}

Identification of differentially expressed lncRNAs and mRNAs. Based on the screening criteria, $\mathrm{FC}>1.2$ and $\mathrm{P}<0.05$, differentially expressed lncRNAs and mRNAs in the GSE67472 dataset were identified. A total of 159 differentially expressed lncRNAs and 1,261 mRNAs were identified. In total, 48 lncRNAs were found to be upregulated and 111 lncRNAs were found to be downregulated while 744 mRNAs were found to be upregulated and 517 mRNAs were found to be downregulated. Hierarchical clustering was conducted to evaluate the altered expression of lncRNAs and mRNAs in the samples (Fig. 1).

Functional annotation and pathway analysis for differentially expressed $m R N A s$. GO and KEGG pathway enrichment analyses were performed to determine the functions of the identified differentially expressed mRNAs. GO analysis revealed that the top 30 terms of the 2,184 and 1,605 GO terms 


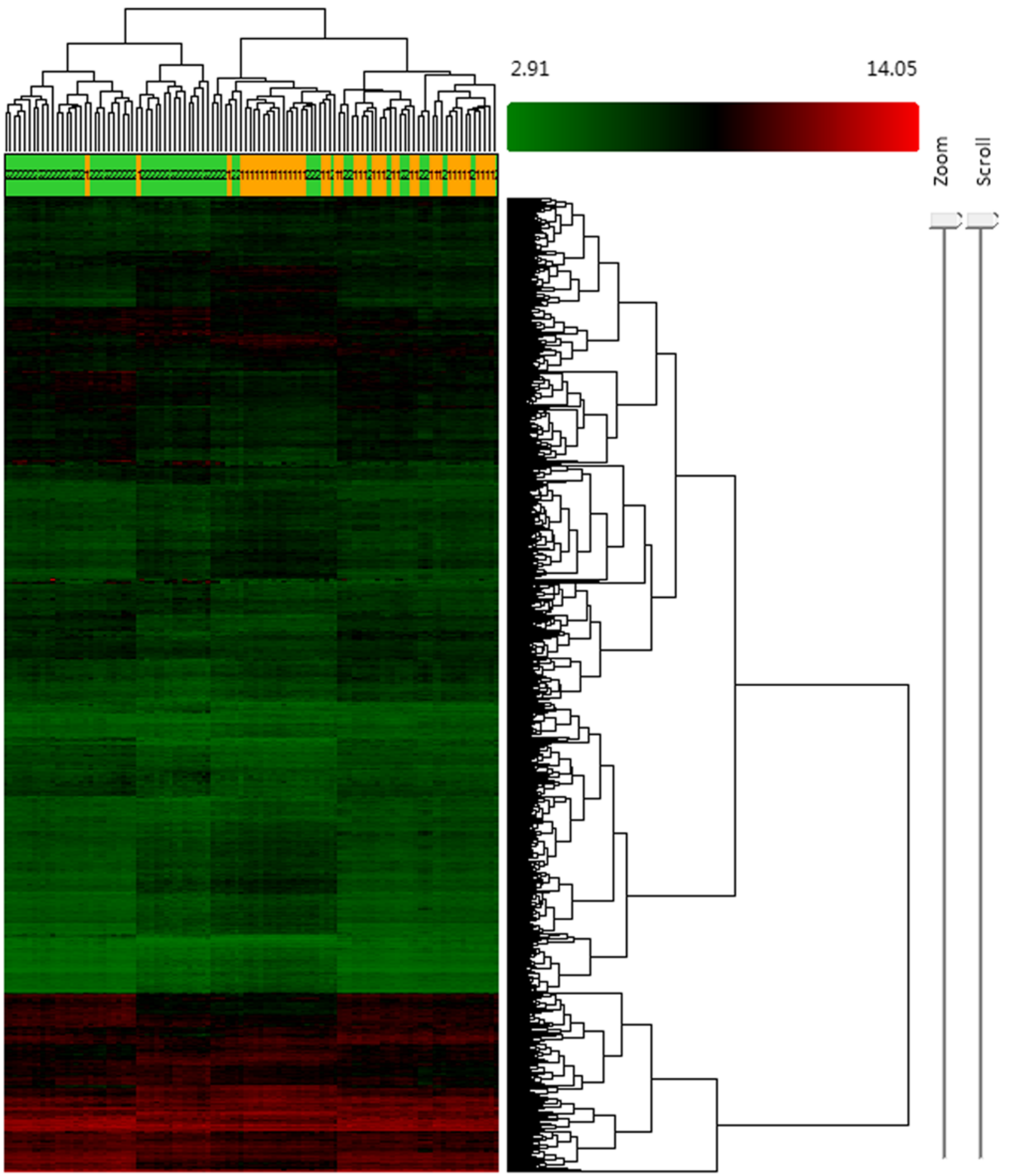

Figure 1. Hierarchical clustering of the differentially expressed lncRNAs and mRNAs. Upregulated expression is presented in red and downregulated expression is presented in green. Each row represents an lncRNA/mRNA probe, and each column represents a tissue sample. 1 (yellow) indicates samples from the healthy control group, 2 (green) indicates samples from the asthma group. lncRNA, long non-coding RNA.

were enriched in the upregulated (Fig. 2A) and downregulated (Fig. 2C) genes, respectively. In the pathway analysis, the top 30 of the 245 and 219 pathways were enriched in the upregulated (Fig. 2B) and downregulated (Fig. 2D) genes, respectively.

PPI network. The PPI network of genes with significantly altered expression $(\mathrm{FC}>1.2$ and $\mathrm{P}<0.05)$ was delineated using the STRING database. The PPI network contained 421 nodes and 1,232 edges (Fig. 3). Genes with the top 15 highest degrees are presented in Table I. Genes with a high degree may be potential targets for clinical treatment (32).

Construction of the IncRNA-mRNA WGCNA. The IncRNAmRNA co-expression network was established to investigate 
Table I. Top 15 genes with the connectivity in the protein-protein interaction network.

\begin{tabular}{|c|c|c|c|c|}
\hline Gene & Node degree & Fold change (asthma/control) & P-value & Trend \\
\hline AKT1 & 55 & 1.24 & $3.13 \times 10^{-4}$ & Up \\
\hline $\mathrm{ACACB}$ & 49 & 1.27 & $1.71 \times 10^{-3}$ & Down \\
\hline DNAJC10 & 37 & 1.36 & $2.09 \times 10^{-2}$ & Up \\
\hline FOS & 35 & 1.37 & $6.04 \times 10^{-3}$ & Down \\
\hline GART & 35 & 1.43 & $3.91 \times 10^{-4}$ & Down \\
\hline LRGUK & 33 & 1.25 & $3.20 \times 10^{-3}$ & Down \\
\hline INSR & 31 & 1.44 & $1.00 \times 10^{-5}$ & Down \\
\hline KIT & 28 & 1.62 & $2.25 \times 10^{-7}$ & Up \\
\hline ENO2 & 26 & 1.22 & $1.75 \times 10^{-3}$ & Down \\
\hline HSPA5 & 25 & 1.33 & $1.33 \times 10^{-2}$ & Up \\
\hline CD44 & 21 & 1.62 & $4.30 \times 10^{-5}$ & Up \\
\hline DYNC2H1 & 21 & 1.33 & $4.00 \times 10^{-5}$ & Down \\
\hline EGR1 & 21 & 1.31 & $4.19 \times 10^{-2}$ & Down \\
\hline HDAC9 & 21 & 1.66 & $1.59 \times 10^{-7}$ & $\mathrm{Up}$ \\
\hline HPGDS & 21 & 1.48 & $2.00 \times 10^{-5}$ & $\mathrm{Up}$ \\
\hline
\end{tabular}

Up, upregulated; down, downregulated.

A

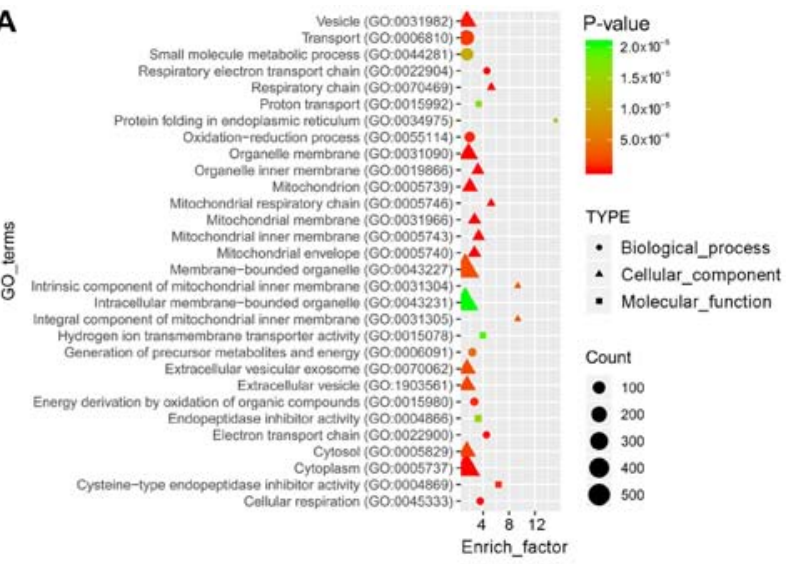

C

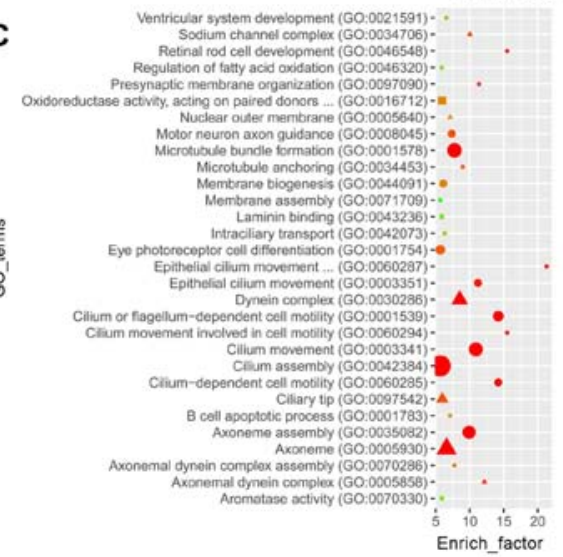

Count

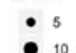

10

${ }^{15}$

P-value

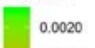

0.0020
0.0015

0.0010

0.0005

TYPE

- Biological_process

- Cellular_component

- Molecular_function
B

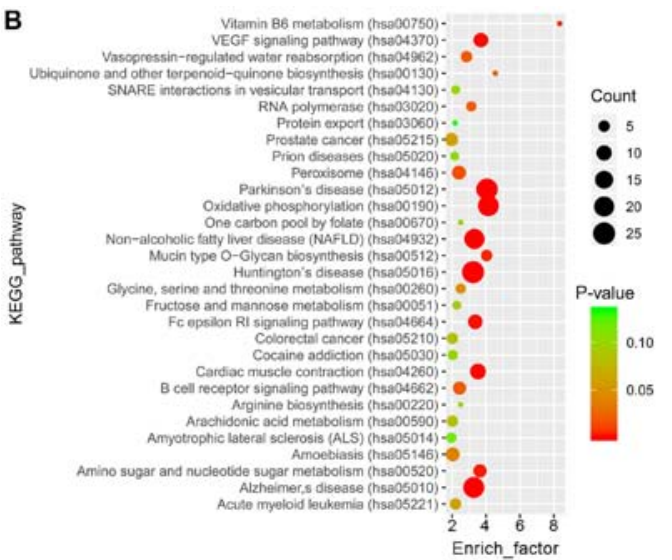

D

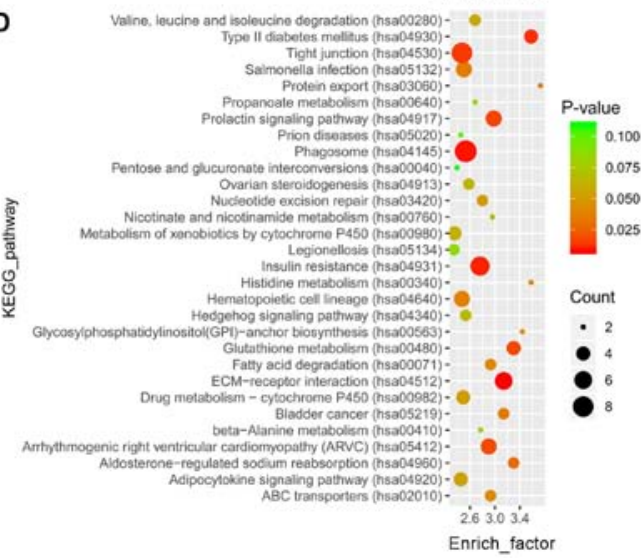

Figure 2. Function annotation and pathway analysis for differentially expressed mRNAs. The top 30 GO enrichment terms of the (A) up- and (C) downregulated genes are presented. The top 30 KEGG pathway terms from enrichment analysis of the (B) up- and (D) downregulated genes are presented. The enrichment factor refers to the ratio of the number of genes in the GO/KEGG entry following enrichment to the total number of genes in the GO/KEGG entry. An increased enrichment factor indicates greater enrichment. Lower P-values indicate higher significance. GO, Gene Ontology; KEGG, Kyoto Encyclopedia of Genes and Genomes.

the association between differentially expressed lncRNAs and mRNAs. In the present study, a cluster dendrogram was obtained using the WGCNA package in R (Fig. 4A); two weighted co-expression subnetworks were identified. lncRNA-mRNA weighted co-expression networks were generated based on genes with the top 30 degrees in the two 


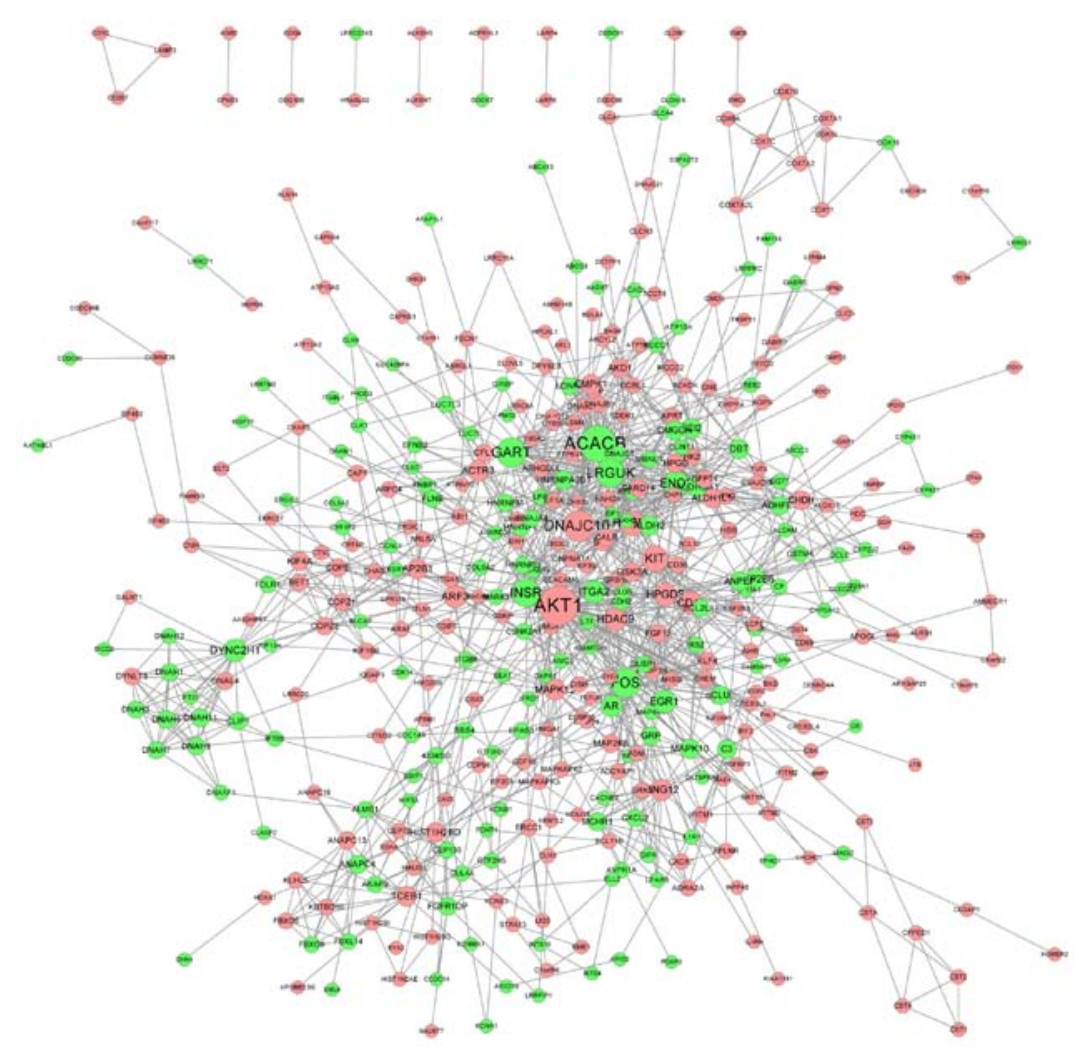

Figure 3. PPI network of the differentially expressed genes constructed with Cytoscape software. Red nodes represent upregulated genes, while green nodes represent downregulated genes. The edges indicated the association between genes. The degree of one gene represents the number of interactions with other genes. The larger the node, the higher the connectivity. A high connectivity indicates the importance of a gene in the PPI network. PPI, protein-protein interaction.

A A

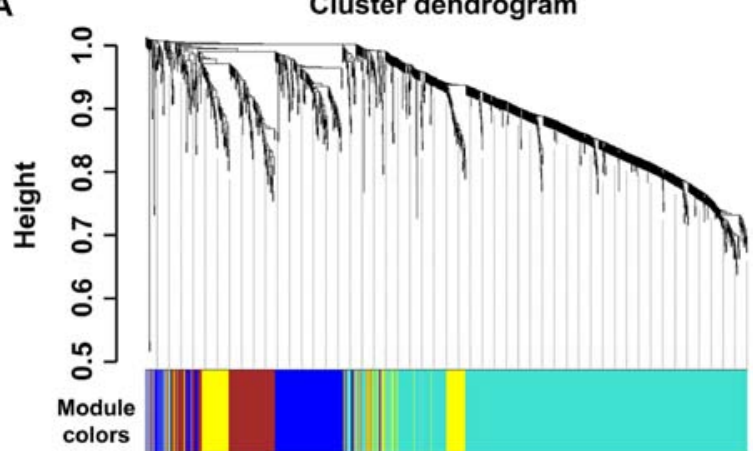

B
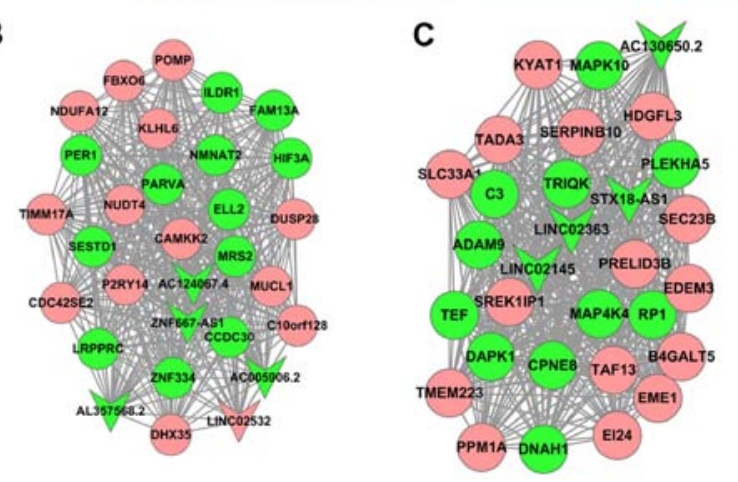

C

Figure 4. Construction of the lncRNA-mRNA weighted co-expression network. (A) Cluster dendrogram. The subnetwork of genes with the top 30 connectivity degrees in (B) the blue module and (C) the turquoise module. The gene dendrogram indicated the co-expression modules defined by the weighted correlation network analysis. Red and green arrow heads represent up- and downregulated lncRNAs, respectively. Up- and downregulated mRNAs were represented by red and green circles, respectively. lncRNA, long non-coding RNA. modules, which comprised 8 lncRNAs and 52 mRNAs. The two modules included a number of IncRNA-mRNA co-expression interactions, which indicated the mRNAs that could be regulated by certain IncRNAs. The IncRNAs in the blue and turquoise modules are listed in Table II. As presented in Fig. 4B and C, the round and arrow-shaped nodes represented mRNAs and lncRNAs, respectively; red indicated upregulation, while green indicated downregulation expression.

Functional annotation and pathway analysis for the differentially expressed mRNAs in the blue and turquoise modules. GO analysis revealed a total of 756 and 1,882 enriched terms in the blue and the turquoise modules, respectively. The top 30 terms are presented in Fig. 5A and C. KEGG analysis revealed that 116 and 164 pathways were enriched in the two modules, respectively. The top 30 pathways are presented in Fig. 5B and D.

\section{Discussion}

Asthma is a common health issue, which poses an economic and social burden to patients (2); however, the pathogenesis of this condition remains poorly understood. To investigate the pathogenesis of asthma and to identify potential biomarkers for clinical treatment in the present study, IncRNA and mRNA expression data in the GSE67472 dataset were downloaded. A total of 159 and 1,261 dysregulated lncRNAs and mRNAs, respectively, were identified in bronchial mucosa samples obtained from patients with asthma compared with samples obtained from normal controls. 

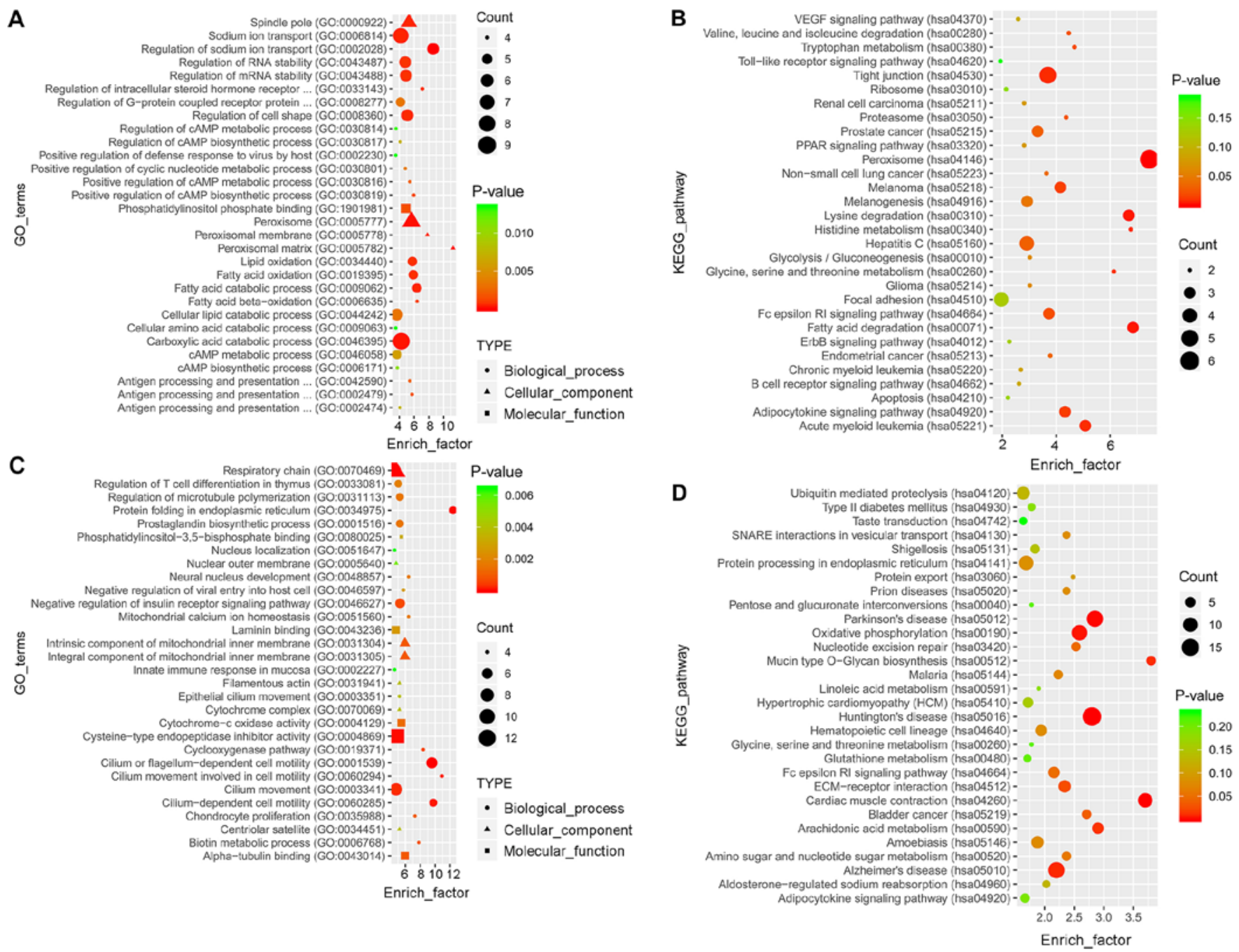

Figure 5. Functional annotation and pathway analysis for the differentially expressed mRNAs in the blue and turquoise modules. The top $30 \mathrm{GO}$ annotations of the differentially expressed mRNAs in (A) the blue module and (C) the turquoise module are presented. The top 30 KEGG pathways from enrichment analysis in (B) the blue module and (D) the turquoise module are presented. The enrichment factor refers to the ratio of the number of genes located in the GO/KEGG entry following enrichment to the total number of genes in the GO/KEGG entry. An increased rich factor indicates greater enrichment. Lower P-values indicate higher significance. GO, gene ontolgy; KEGG, Kyoto Encyclopedia of Genes and Genomes.

Table II. IncRNAs in the blue module and the turquoise module.

\begin{tabular}{llccc}
\hline lncRNA & Module & Trend & P-value & Fold change \\
\hline AC124067.4 & Blue & Down & $1.99 \times 10^{-2}$ & 1.31 \\
ZNF667-AS1 & Blue & Down & $4.80 \times 10^{-2}$ & 1.24 \\
AC005906.2 & Blue & Down & $2.28 \times 10^{-2}$ & 1.26 \\
AL357568.2 & Blue & Down & $6.92 \times 10^{-4}$ & 1.22 \\
AC130650.2 & Turquoise & Down & $9.64 \times 10^{-7}$ & 1.31 \\
STX18-AS1 & Turquoise & Down & $8.91 \times 10^{-4}$ & 1.24 \\
LINC02363 & Turquoise & Down & $1.34 \times 10^{-2}$ & 1.22 \\
LINC02145 & Turquoise & Down & $2.00 \times 10^{-6}$ & 1.37 \\
\hline
\end{tabular}

lncRNA, long non-coding RNA; down, downregulated.

Genes and their protein products are the basic components of cells, and can be assembled into functional modules.
Gene co-expression networks are used to investigate the associations between gene transcripts (33). WGCNA is a biological application for screening clusters (modules) of highly associated genes and demonstrates the link between genes across microarray samples. This analytic tool has been utilized in numerous studies $(12,18,29,34)$. Using WGCNA and bioinformatics analysis, the major biological functions of asthma-associated lncRNAs, and the potential underlying molecular mechanisms in which these IncRNAs may be involved, were investigated in the present study. WGCNA analysis identified 8 key lncRNAs and 52 genes in two modules; genes in the blue module were enriched in the GOs of several metabolic and catabolic processes. Previous studies have indicated that lncRNAs are involved in asthma $(35,36)$. ZNF667 antisense RNA 1 (ZNF667-AS1) inhibits the inflammatory response of spinal cord injury by suppressing the Janus kinase-STAT pathway (37-41). The present study observed that the level of lncRNA ZNF667-AS1 in asthma was downregulated in comparison with healthy controls. Therefore, IncRNA ZNF667-AS1 may play a role in the pathogenesis of asthma. 
Airway remodeling plays an important role in the progressive worsening of asthma and is irreversible $(1,2)$. Vascular endothelial growth factor $(42,43)$, oxidative stress $(44,45)$, the Fc $\varepsilon$ RI signaling pathway $(46,47)$, and the dysregulation of amino acids, sugar and nucleotide metabolism $(48,49)$ have been implicated in asthma. Functional gene analysis of the 159 lncRNAs and 1,261 mRNAs identified in the present study demonstrated that the aforementioned pathways are involved in the pathogenesis of asthma. In order to investigate the role of the 8 key lncRNAs and 52 genes in two modules, functional analysis using GO and KEGG was conducted. Certain pathways, including 'Toll-like receptors' $(50,51)$, 'activation of PPAR signaling pathway' (52) and 'eosinophil apoptosis' (53), are important for elucidating the mechanisms underlying the pathogenesis of asthma. Genes in the turquoise module were enriched in the following GO terms: 'respiratory chain', 'regulation of T cell differentiation in thymus' and 'cilium movement involved in cell motility'. KEGG analysis revealed that genes in the turquoise module were enriched in the 'Fc \& RI signaling pathway' and 'ECM-receptor interaction'. These results indicated that genes in the two modules may serve key roles in the initiation and development of asthma.

In the present study, upregulated protein kinase B (Akt) exhibited the highest degree in the PPI network and was significantly enriched in the 'VEGF signaling pathway' and 'Fc $\varepsilon$ RI signaling pathway'. Akt is also involved in the phosphoinositide 3-kinase/Akt signaling pathway, which served a key role in lung inflammation and airway remodeling in a rat model of ovalbumin (OVA)-induced asthma (54). The expression of phosphorylated-Akt was increased in the lung tissues of rats with OVA-induced asthma compared with controls.

IncRNAs regulate the transcription and expression of mRNAs, and participate in the initiation and development of various diseases $(7,55)$. Therefore, the lncRNAs identified in the present study and their interacting genes may serve important roles in the onset and development of asthma. There are some limitations to the present study. The data was only extracted from one dataset that was not confirmed in independent studies. Additionally, cell, animal and clinical experiments are required to corroborate the results obtained in the present study as the mRNA expression level may not always be consistent with the protein level (56). Furthermore, the interactions between the identified lncRNA and mRNA requires experimental verification. Such future research may elucidate the mechanisms underlying lncRNAs and mRNAs in the development of asthma.

\section{Acknowledgements}

The authors would like to thank Mr Qiang Fan (Ao Ji Bio-tech Co., Ltd.) for assisting with the data analysis.

\section{Funding}

The present study was supported by the National Natural Science Foundation of China (grant no. 81804043).

\section{Availability of data and materials}

The datasets analyzed during the present study are available from the Gene Expression Omnibus repository, www.ncbi. nlm.nih.gov/geo/query/acc.cgi?acc=GSE67472.

\section{Authors' contributions}

JG conceived and designed the study. XL and YZ acquired, analyzed and interpreted data and wrote the manuscript. NJ and $\mathrm{HJ}$ analyzed data and critically revised the manuscript. JG generally supervised the research group and gave final approval. All authors have read and approved the manuscript.

\section{Ethics approval and consent to participate}

Not applicable.

\section{Patient consent for publication}

Not applicable.

\section{Competing interests}

The authors declare that they have no competing interests.

\section{References}

1. Abreu SC, Lopes-pacheco M, da Silva AL, Xisto DG, de Oliveira TB, Kitoko JZ, de Castro LL, Amorim NR, Martins V, Silva LHA, et al: Eicosapentaenoic acid enhances the effects of mesenchymal stromal cell therapy in experimental allergic asthma. Front Immunol 9: 1147, 2018.

2. Mejias SG and Ramphul K: Prevalence and associated risk factors of bronchial asthma in children in santo domingo, dominican republic. Cureus 10: e2211, 2018.

3. Soriano JB, Abajobir AA, Abate KH, Abera SF, Agrawal A, Ahmed MB, Aichour AN, Aichour I, Aichour MT, Alam K, et al: Global, regional, and national deaths, prevalence, disability-adjusted life years, and years lived with disability for chronic obstructive pulmonary disease and asthma, 1990-2015: A systematic analysis for the Global Burden of Disease Study 2015. Lancet Respir Med 5: 691-706, 2017.

4. de Castro LL, Xisto DG, Kitoko JZ, Cruz FF, Olsen PC, Redondo PAG,Ferreira TPT, Weiss DJ, Martins MA, Morales MM and Rocco PRM: Human adipose tissue mesenchymal stromal cells and their extracellular vesicles act differentially on lung mechanics and inflammation in experimental allergic asthma. Stem Cell Res Ther 8: 151-162, 2017.

5. Ponting CP, Oliver PL and Reik W: Evolution and functions of long noncoding RNAs. Cell 136: 629-641, 2009.

6. Mercer TR, Dinger ME and Mattick JS: Long non-coding RNAs: Insights into functions. Nat Rev Genet 10: 155-159, 2009.

7. Zhang ZK, Li J, Guan D, Liang C, Zhuo Z, Liu J, Lu A, Zhang G and Zhang BT: A newly identified lncRNA MAR1 acts as a miR-487b sponge to promote skeletal muscle differentiation and regeneration. J Cachexia Sarcopenia Muscle 9: 613-626, 2018.

8. Li XQ, Ren ZX, Li K, Huang JJ, Huang ZT, Zhou TR, Cao HY, Zhang FX and Tan B: Key Anti-fibrosis associated long noncoding RNAs identified in human hepatic stellate cell via transcriptome sequencing analysis. Int J Mol Sci 19: E675, 2018.

9. Yan B, Liu JY, Yao J, Li XM, Wang XQ, Li YJ, Tao ZF, Song YC, Chen Q and Jiang Q: IncRNA-MIAT regulates microvascular dysfunction by functioning as a competing endogenous RNA. Circ Res 116: 1143-1156, 2015.

10. Wang K, Liu CY, Zhou LY, Wang JX, Wang M, Zhao B, Zhao WK, Xu SJ, Fan LH, Zhang XJ, et al: APF lncRNA regulates autophagy and myocardial infarction by targeting miR-188-3p. Nat Commun 6: 6779, 2015.

11. Xing Z, Park PK, Lin C and Yang L: LncRNA BCAR4 wires up signaling transduction in breast cancer. RNA Biol 12: 681-689, 2015.

12. Gao JR, Qin XJ, Jiang H, Gao YC, Guo MF and Jiang NN: Potential role of IncRNAs in contributing to pathogenesis of chronic glomerulonephritis based on microarray data. Gene 643: 46-54, 2018.

13. Barrett T, Wilhite SE, Ledoux P, Evangelista C, Kim IF, Tomashevsky M, Marshall KA, Phillippy KH, Sherman PM, Holko M, et al: NCBI GEO: Archive for functional genomics data sets-update. Nucleic Acids Res 41 (Database): D991-D995, 2013. 
14. Sharma M, Batra J , Mabalirajan U, Sharma S, Nagarkatti R, Aich J, Sharma SK, Niphadkar PV and Ghosh B: A genetic variation in inositol polyphosphate 4 phosphatase a enhances susceptibility to asthma. Am J Respir Crit Care Med 177: 712-719, 2008.

15. Christenson SA, Steiling K, van den Berge M, Hijazi K, Hiemstra PS,Postma DS, Lenburg ME, Spira A and WoodruffPG: Asthma-COPD overlap. Clinical relevance of genomic signatures of type 2 inflammation in chronic obstructive pulmonary disease. Am J Respir Crit Care Med 191: 758-766, 2015

16. Christenson SA, Steiling K, van den Berge M, Hijazi K, Hiemstra PS, Postma DS, Lenburg ME, Spira A and Woodruff PG: Asthma-COPD Overlap: Clinical Relevance of Genomic Signatures of Type 2 Inflammation in COPD. Am J Respir Crit Care Med 191: 758-766, 2015.

17. Zhang XQ, Sun S, Pu JK, Tsang AC, Lee D, Man VO, Lui WM, Wong ST and Leung GK: Long non-coding RNA expression profiles predict clinical phenotypes in glioma. Neurobiol Dis 48: 1-8, 2012.

18. Yu C, Ni HJ, Zhao YC, Chen K, Li M, Li C, Zhu XD and Fu Q: Potential role of lncRNAs in contributing to pathogenesis of intervertebral disc degeneration based on microarray data. Med Sci Monit 21: 3449-3458, 2015.

19. Luchessi AD, Silbiger VN, Hirata RD, Lima-Neto LG, Cavichioli D, Iñiguez A, Bravo M, Bastos G, Sousa AG, Brión M, et al: Pharmacogenomics of anti-platelet therapy focused on peripheral blood cells of coronary arterial disease patients. Clin Chim Acta 425: 9-17, 2013.

20. Xing ZH, Chu C, Chen L and Kong XY: The use of Gene Ontology terms and KEGG pathways for analysis and prediction of oncogenes. Biochim Biophys Acta 1860: 2725-2734, 2016.

21. Maere S, Heymans K and Kuiper M: BiNGO: A Cytoscape plugin to assess overrepresentation of Gene Ontology categories in Biological Networks. Bioinformatics 21: 3448-3449, 2005.

22. Huang da W, Sherman BT and Lempicki RA: Systematic and integrative analysis of large gene lists using DAVID bioinformatics resources. Nat Protoc 4: 44-57, 2009.

23. Huang DW, Sherman BT and Lempicki RA: Bioinformatics enrichment tools: Paths toward the comprehensive functional analysis of large gene lists. Nucleic Acids Res 37: 1-13, 2009.

24. von Mering C, Jensen LJ, Snel B, Hooper SD, Krupp M, Foglierini M, Jouffre N, Huynen MA and Bork P: STRING: Known and predicted protein-protein associations, integrated and transferred across organisms. Nucleic Acids Res 33: D433-D437, 2005.

25. Cuesta-Astroz Y, Santos A, Oliveira G and Jensen LJ: Analysis of predicted host-parasite interactomes reveals commonalities and specificities related to parasitic lifestyle and tissues tropism. Front Immunol 10: 212, 2019.

26. Wang Y, Ruan Z, Yu S, Tian T, Liang X, Jing L, Li W, Wang X, Xiang L, Claret FX, et al: A four-methylated mRNA signature-based risk score system predicts survival in patients with hepatocellular carcinoma. Aging (Albany NY) 11: 160-173, 2019.

27. Safari-Alighiarloo N, Taghizadeh M, Tabatabaei SM, Shahsavari S, Namaki S, Khodakarim S and Rezaei-Tavirani M: Identification of new key genes for type 1 diabetes through construction and analysis of protein-protein interaction networks based on blood and pancreatic islet transcriptomes. J Diabetes 9: 764-777, 2017.

28. Le DH and Pham VH: HGPEC: A Cytoscape app for prediction of novel disease-gene and disease-disease associations and evidence collection based on a random walk on heterogeneous network. Bmc Syst Biol 11: 61, 2017.

29. Langfelder $P$ and Horvath S: WGCNA: An R package for weighted correlation network analysis. Bmc Bioinformatics 9: 559, 2008.

30. Zhang B and Horvath S: A general framework for weighted gene co-expression network analysis. Stat Appl Genet Mol Biol 4: Article 17, 2005

31. Dudoit $\mathrm{S}$ and Fridlyand J: A prediction-based resampling method for estimating the number of clusters in a dataset. Genome Biol 3 : RESEARCH0036, 2002.

32. Hsin KY, Ghosh S and Kitano H: Combining machine learning systems and multiple docking simulation packages to improve docking prediction reliability for network pharmacology. PLoS One 8: e83922, 2013.

33. Langfelder $P$ and Horvath $S$ : Eigengene networks for studying the relationships between co-expression modules. Bmc Syst Biol 1: 54, 2007.

34. Zhao W, Langfelder P, Fuller T, Dong J, Li A and Hovarth S: Weighted gene coexpression network analysis: State of the art. J Biopharm Stat 20: 281-300, 2010.

35. Wang SY, Fan XL, Yu QN, Deng MX, Sun YQ, Gao WX, Li CL, Shi JB and Fu QL: The lncRNAs involved in mouse airway allergic inflammation following induced pluripotent stem cell-mesenchymal stem cell treatment. Stem Cell Res Ther 8: 2, 2017.
36. Zhang XY, Zhang LX, Tian CJ, Tang XY, Zhao LM, Guo YL, Cheng DJ, Chen XL, Ma LJ and Chen ZC: LncRNAs BCYRN1 promoted the proliferation and migration of rat airway smooth muscle cells in asthma via upregulating the expression of transient receptor potential 1. Am J Transl Res 8: 3409-3418, 2016.

37. Vrba L, Garbe JC, Stampfer MR and Futscher BW: A lincRNA connected to cell mortality and epigenetically-silenced in most common human cancers. Epigenetics 10: 1074-1083, 2015.

38. Meng W, Cui W, Zhao L, Chi W, Cao H and Wang B: Aberrant methylation and downregulation of ZNF667-AS1 and ZNF667 promote the malignant progression of laryngeal squamous cell carcinoma. J Biomed Sci 26: 13, 2019.

39. Li JW, Kuang Y, Chen L and Wang JF: LncRNA ZNF667-AS1 inhibits inflammatory response and promotes recovery of spinal cord injury via suppressing JAK-STAT pathway. Eur Rev Med Pharmacol Sci 22: 7614-7620, 2018.

40. Zhao LP, Li RH, Han DM, Zhang XQ, Nian GX, Wu MX, Feng Y, Zhang L and Sun ZG: Independent prognostic Factor of low-expressed LncRNA ZNF667-AS1 for cervical cancer and inhibitory function on the proliferation of cervical cancer. Eur Rev Med Pharmacol Sci 21: 5353-5360, 2017.

41. Vrba L and Futscher BW: Epigenetic silencing of 1ncRNA MORT in 16 TCGA cancer types. F1000Res 7: 211, 2018.

42. Smith R: Is VEGF a potential therapeutic target in asthma? Pneumologia 63: 194, 197-199, 2014.

43. Na HJ, Hwang JY, Lee KS, Choi YK, Choe J, Kim JY, Moon HE, Kim KW, Koh GY, Lee H, et al: TRAIL negatively regulates VEGF-induced angiogenesis via caspase-8-mediated enzymatic and non-enzymatic functions. Angiogenesis 17: 179-194, 2014.

44. Lan N, Luo G, Yang X, Cheng Y, Zhang Y, Wang X, Wang X, Xie T, Li G, Liu Z and Zhong N: 25-Hydroxyvitamin D3-deficiency enhances oxidative stress and corticosteroid resistance in severe asthma exacerbation. PLoS One 9: e111599, 2014.

45. Chung KF and Marwick JA: Molecular mechanisms of oxidative stress in airways and lungs with reference to asthma and chronic obstructive pulmonary disease. Ann N Y Acad Sci 1203: 85-91, 2010.

46. Wu LC: Immunoglobulin E receptor signaling and asthma. J Biol Chem 286: 32891-32897, 2011.

47. Gounni AS, Wellemans V, Yang J, Bellesort F, Kassiri K, Gangloff S, Guenounou M, Halayko AJ, Hamid Q and Lamkhioued B: Human airway smooth muscle cells express the high affinity receptor for $\mathrm{IgE}$ ( $\mathrm{Fc}$ epsilon RI): A critical role of Fc epsilon RI in human airway smooth muscle cell function. J Immunol 175: 2613-2621, 2005.

48. Ho WE, Xu YJ, Xu F, Cheng C, Peh HY, Tannenbaum SR, Wong WS and Ong CN: Metabolomics reveals altered metabolic pathways in experimental asthma. Am J Resp Cell Mol 48: 204-211, 2013.

49. Xu W, Comhair SAA, Janocha AJ, Lara A, Mavrakis LA, Bennett CD, Kalhan SC and Erzurum SC: Arginine metabolic endotypes related to asthma severity. PLoS One 12: e0183066, 2017.

50. Xiao HT, Liao Z, Chen L and Tong RS: A promising approach for asthma treatment by multiwayly modulating toll-like receptors. Eur Rev Med Pharmacol Sci 16: 2088-2091, 2012.

51. Bezemer GF, Sagar S, ven Bergenhenegouwen J, Georgiou NA, Garssen J, Kraneveld AD and Folkerts G: Dual role of Toll-like receptors in asthma and chronic obstructive pulmonary disease. Pharmcol Rev 64: 337-358, 2012.

52. Xu J, Zhu YT, Wang GZ, Han D, Wu YY, Zhang DX, Liu Y, Zhang YH, Xie XM, Li SJ, et al: The PPAR $\gamma$ agonist, rosglitazone, attenuates airway inflammation and remodeling via heme oxygenase-1 in murine model of asthma. Acta Pharmacol Sin 36: 171-178, 2015.

53. Ilmarinen $\mathrm{P}$ and Kankaanranta $\mathrm{H}$ : Eosinophil apoptosis as a therapeutic target in allergic asthma. Basic Clin Pharmacol Toxicol 114: 109-117, 2014.

54. Lin HY, Xu L, Xie SS, Yu F, Hu HY, Song XL and Wang CH: Mesenchymal stem cells suppress lung inflammation and airway remodeling in chronic asthma rat model via PI3K/Akt signaling pathway. Int J Clin Exp Pathol 8: 8958-8967, 2015.

55. Kung JT, Colognori D and Lee JT: Long noncoding RNAs: Past, present, and future. Genetics 193: 651-669, 2013.

56. Huang KL, Li S, Mertins P, Cao S, Gunawardena HP, Ruggles KV, Mani DR, Clauser KR, Tanioka M, Usary J, et al: Proteogenomic integration reveals therapeutic targets in breast cancer xenografts. Nat Commun 8: 14864, 2017.

This work is licensed under a Creative Commons Attribution-NonCommercial-NoDerivatives 4.0 International (CC BY-NC-ND 4.0) License. 